\title{
Maximum Energy Sequential Matrix Diagonalisation for Parahermitian Matrices
}

\author{
Jamie Corr*, Keith Thompson*, Stephan Weiss*, John G. McWhirter ${ }^{\dagger}$, Ian K. Proudler ${ }^{\ddagger}$ \\ *Department of Electronic \& Electrical Engineering, University of Strathclyde, Glasgow, Scotland \\ ${ }^{\dagger}$ School of Engineering, Cardiff University, Cardiff, Wales, UK \\ ${ }_{\ddagger}^{\ddagger}$ School of Electrical, Electronics and Systems Engineering, Loughborough University, Loughborough, UK \\ \{jamie.corr,keith.thompson,stephan.weiss\}@ strath.ac.uk
}

\begin{abstract}
Sequential matrix diagonalisation (SMD) refers to a family of algorithms to iteratively approximate a polynomial matrix eigenvalue decomposition. Key is to transfer as much energy as possible from off-diagonal elements to the diagonal per iteration, which has led to fast converging SMD versions involving judicious shifts within the polynomial matrix. Through an exhaustive search, this paper determines the optimum shift in terms of energy transfer. Though costly to implement, this scheme yields an important benchmark to which limited search strategies can be compared. In simulations, multiple-shift SMD algorithms can perform within $10 \%$ of the optimum energy transfer per iteration step.
\end{abstract}

\section{INTRODUCTION}

Polynomial matrices arise in many broadband multichannel or array problems, where a multivariate process, described by $\mathbf{x}[n] \in \mathbb{C}^{M}$, requires the use of delays rather than phase shifts which leads to a space-time covariance matrix $\mathbf{R}[\tau]=\mathcal{E}\left\{\mathbf{x}[n] \mathbf{x}^{\mathrm{H}}[n-\tau]\right\}$, with $\mathcal{E}\{\cdot\}$ being the expectation operator. Its z-transform, the cross-spectral density matrix $\boldsymbol{R}(z)=\sum_{\tau} \mathbf{R}[\tau] z^{-\tau} \bullet-\circ \mathbf{R}[\tau]$, is a polynomial in $z[1]$ and possesses the parahermitian property $\boldsymbol{R}(z)=\tilde{\boldsymbol{R}}(z)$, where $\tilde{\boldsymbol{R}}(z)=\boldsymbol{R}^{\mathrm{H}}\left(z^{-1}\right)$ is the parahermitian of $\mathbf{R}(z)$.

A polynomial EVD (PEVD) of $\mathbf{R}(z)$ is required to extend the EVD's utility from many narrowband problems to the broadband case [2],

$$
\boldsymbol{R}(z) \approx \boldsymbol{Q}(z) \boldsymbol{D}(z) \tilde{\boldsymbol{Q}}(z),
$$

with $\boldsymbol{Q}(z)$ paraunitary, i.e. $\boldsymbol{Q}(z) \tilde{\boldsymbol{Q}}(z)=\mathbf{I}$, and a diagonal $\boldsymbol{D}(z)$,

$$
\boldsymbol{D}(z)=\operatorname{diag}\left\{D_{0}(z) D_{1}(z) \ldots D_{M-1}(z)\right\} \quad .
$$

Similar to an ordered EVD or an SVD [3], the polynomial eigenvalues in $\boldsymbol{D}(z)$ are ordered, such that the power spectral densities $D_{m}\left(e^{j \Omega}\right)=\left.D_{m}(z)\right|_{z=e^{j \Omega}}$ satisfy

$$
D_{m+1}\left(e^{j \Omega}\right) \geq D_{m}\left(e^{j \Omega}\right) \forall \Omega m=0 \ldots(M-1) .
$$

Equality in (1) for FIR paraunitary matrices is not guaranteed [2], but is likely to be valid for high orders of $\mathbf{Q}(z)$ in close approximation [4].

The PEVD is important for a number of applications. This includes subband coding [5], filter bank-based channel coding [6], design of broadband precoding and equalisation of MIMO systems [7], and broadband angle of arrival estimation [8], to name but a few. A number of these methods utilise broadband subspace techniques [6]-[8], where the accuracy of the PEVD w.r.t. diagonalisation and spectral majorisation is directly linked to the performance of the specific applications.

A number of iterative algorithms to approximate (1) have been developed, including the second order sequential best rotation (SBR2) algorithm [2] and a coding gain optimised SBR2 version [5]. SBR2 has been successful at transferring the energy of the maximum off-diagonal element at every step, and has been proven to converge. Iterations will stop once offdiagonal elements are suppressed below a given threshold. An approximate PEVD (APEVD) [9] is based on a fixed target order for the decomposition, with convergence not proven. A recent sequential matrix diagonalisation (SMD, [10]) algorithm clears an entire column per iteration; by transferring more energy, SMD converges faster and to a more accurate diagonalisation than SBR2.

Based on the diagonalisation idea of SMD, a multipleshift (MS-SMD) version has been developed [11], which does not transfer the energy of one but of several columns at every step. This calls for little extra complexity over SMD, but leads to a further enhancement of energy transfer and diagonalisation accuracy. The added complexity lies in the identification of appropriate column shifts. Since each column shift is combined with a shift of the associated row, elements cannot be moved in isolation, and may be affected by a number of subsequent shifts. Therefore, the identification of elements and careful shifting of columns such that no previous effort is undone is tricky and requires a limitation of the search space for the shift parameters. As a further restriction, the extracted $\boldsymbol{Q}(z)$ can be constrained to be causal [12].

Since it is difficult to assess how much the search space for MS-SMD algorithms limits performance, the aim of this paper is to determine the maximum possible energy transfer per iteration step. At present, the only available means is an exhaustive search, which is very expensive but can serve as an important benchmark to which reduced search space methods can be compared.

Below, Sec. II reviews iterative PEVD algorithms of the SMD family. Sec. III outlines the exhaustive search, with results presented in Sec. IV and conclusions drawn in Sec. V. 


\section{ITERATIVE POLYNOMIAL EVD APPROXIMATION Using Sequential Matrix Diagonalisation}

\section{A. General SMD Approach}

All SMD algorithms are initialised with the space-time covariance matrix $\boldsymbol{R}(z)$, such that

$$
\boldsymbol{S}^{(0)}(z)=\mathbf{Q}^{(0) \mathrm{H}} \boldsymbol{R}(z) \mathbf{Q}^{(0)} .
$$

The matrix $\mathbf{Q}^{(0)}$ is the modal matrix obtained from an EVD of $\mathbf{R}[0]=\mathbf{Q}^{(0)} \mathbf{S}^{(0)}[0] \mathbf{Q}^{(0) \mathrm{H}}$, with $\mathbf{S}^{(0)}[\tau] \circ \longrightarrow \boldsymbol{S}^{(0)}(z)$. This ensures that at the start of the iteration, the lag zero or instantaneous covariance matrix $\mathbf{S}^{(0)}[0]$ is diagonal.

At the $i$ th iteration, in a first step the SMD family of algorithms perform a shift operation

$$
\boldsymbol{S}^{(i) \prime}(z)=\tilde{\boldsymbol{\Lambda}}^{(i)}(z) \boldsymbol{S}^{(i-1)}(z) \boldsymbol{\Lambda}^{(i)}(z), \quad i=1 \ldots I,
$$

using a diagonal delay matrix $\boldsymbol{\Lambda}^{(i)}(z)$ that depends on the particular algorithm version and will be defined further below. Its aim is to shift one or several columns of $\boldsymbol{S}^{(i-1)}(z)$ such that large off-diagonal elements are transferred to the zero-lag matrix $\mathbf{S}^{(i) \prime}[0]$. In a second step, the off-diagonal elements in $\mathbf{S}^{(i) \prime}[0]$ are eliminated via

$$
\boldsymbol{S}^{(i)}(z)=\mathbf{Q}^{(i) \mathrm{H}} \boldsymbol{S}^{(i) \prime}(z) \mathbf{Q}^{(i)}
$$

by setting $\mathbf{Q}^{(i)}$ as the modal matrix of the EVD of $\mathbf{S}^{(i) \prime}[0]$, $\mathbf{S}^{(i) \prime}[0]=\mathbf{Q}^{(i)} \mathbf{S}^{(i)}[0] \mathbf{Q}^{(i) \mathrm{H}}$.

The iteration with steps (5) and (6) continues until a set threshold for the maximum off-diagonal element or some norm defined over the off-diagonal elements is reached. The SMD algorithm has been proven to converge, since every step reduces the off-diagonal energy, with a transfer onto the diagonal. Since this transfer is performed by paraunitary operations, the overall energy remains unaltered, and the offdiagonal energy therefore continues to reduce with every iteration.

Once the algorithm has been stopped after $I$ iterations, the approximately diagonalised matrix $\boldsymbol{D}(z)$ in (1) is

$$
\boldsymbol{D}(z) \approx \boldsymbol{S}^{(I)}(z) .
$$

The paraunitary matrix in (1) can be assembled via the delay matrices $\boldsymbol{\Lambda}^{(i)}$ and rotations $\boldsymbol{Q}^{(i)}$,

$$
\boldsymbol{Q}(z) \approx \mathbf{Q}^{(0)} \prod_{i=1}^{I} \mathbf{\Lambda}^{(i)}(z) \mathbf{Q}^{(i)}
$$

Thus, the PEVD is approximated with (7) and (8).

\section{B. Sequential Matrix Diagonalisation (SMD)}

SMD in its basic form [10] identifies in the $i$ th iteration a single column - indicated by its column index $k^{(i)}$ and lag index $\tau^{(i)}$ - with maximum $l_{2}$-norm over its off-diagonal elements,

$$
\left\{k^{(i)}, \tau^{(i)}\right\}=\arg \max _{k, \tau}\left\|\hat{\mathbf{s}}_{k}^{(i-1)}[\tau]\right\|_{2} \quad .
$$

The vector $\hat{\mathbf{s}}_{k}^{(i-1)}[\tau] \in \mathbb{C}^{M-1}$ contains all elements of the $k^{(i)}$ th column at the $\tau^{(i)}$ lag, except for the diagonal element.
With the parameters identified according to (9), the column shift is performed by

$$
\boldsymbol{\Lambda}^{(i)}(z)=\operatorname{diag}\{\underbrace{1 \ldots 1}_{k^{(i)}-1} z^{-\tau^{(i)}} \underbrace{1 \ldots 1}_{M-k^{(i)}}\}
$$

as the delay matrix in (5).

\section{Maximum Element SMD (ME-SMD)}

To ease the search in (9), the costlier $l_{2}$-norm can be replaced by the less expensive $l_{\infty}$-norm, such that the shifted column is identified by containing the maximum off-diagonal element. Even though most of the SMD's computation burden lies in the EVD calculation and the application of the modal matrix at all lag values, the ME-SMD version brings a slight computational advantage over the standard SMD as described in Sec. II-B.

\section{Multiple Shift ME-SMD (MSME-SMD)}

The fact, that the complexity of SMD and ME-SMD algorithms is governed by the application of an EVD to $\mathbf{S}^{(i) \prime}[0]$ and the multiplication of $\boldsymbol{S}^{(i) \prime}(z)$ with a unitary matrix, allows the shifting of several columns onto the lag zero matrix $\mathbf{S}^{(i) \prime}[0]$ at little extra cost. This can enhance the energy transfer of off-diagonal elements onto the main diagonal and lead to a significant increase on convergence speed.

To consider multiple shifts, the delay matrix in (5) takes the form

$$
\Lambda^{(i)}(z)=\operatorname{diag}\left\{z^{-\tau_{0}^{(i)}} z^{-\tau_{1}^{(i)}} \ldots z^{-\tau_{M-1}^{(i)}}\right\} .
$$

Determining the delays $\tau_{m}^{(i)}$ is tricky since column shifts by $\Lambda^{(i)}(z)$ will partially be undone by row shifts applied through $\tilde{\Lambda}^{(i)}(z)$ in (5). Search strategies have been aimed at finding and isolating maximum off-diagonal elements that will be unaffected by other shifts [11]. By reducing the search space, $(M-1)$ off-diagonal local maxima can be identified and shifted, with a further restriction to causality of (11) leading to a causally constrained MSME-SMD (C-MSME-SMD).

\section{MAXIMUM ENERGy SMD Algorithm}

\section{A. Idea}

Different to the limited search strategies of previous SMD algorithms reviewed in Sec. II, the maximum energy SMD approach finds the set of shifts for the delay matrix $\Lambda^{(i)}(z)$ in (11), such that the maximum amount of off-diagonal energy is transferred onto the zero lag $\mathbf{S}^{(i) \prime}[0]$ of the parahermitian matrix $\boldsymbol{S}^{(i) \prime}(z)$ at the $i$ th iteration, where it can be eliminated by (6). Below we focus on the $i$ th iteration, and assume that $\boldsymbol{S}^{(i-1)}(z) \in \mathbb{C}^{M \times M}$ has a support of $2 L+1$, i.e. $\mathbf{S}^{(i-1)}[\tau]=$ $0 \forall|\tau|>L$, from which the optimum $\boldsymbol{\Lambda}^{(i)}(z)$ can be determined via an exhaustive search. 


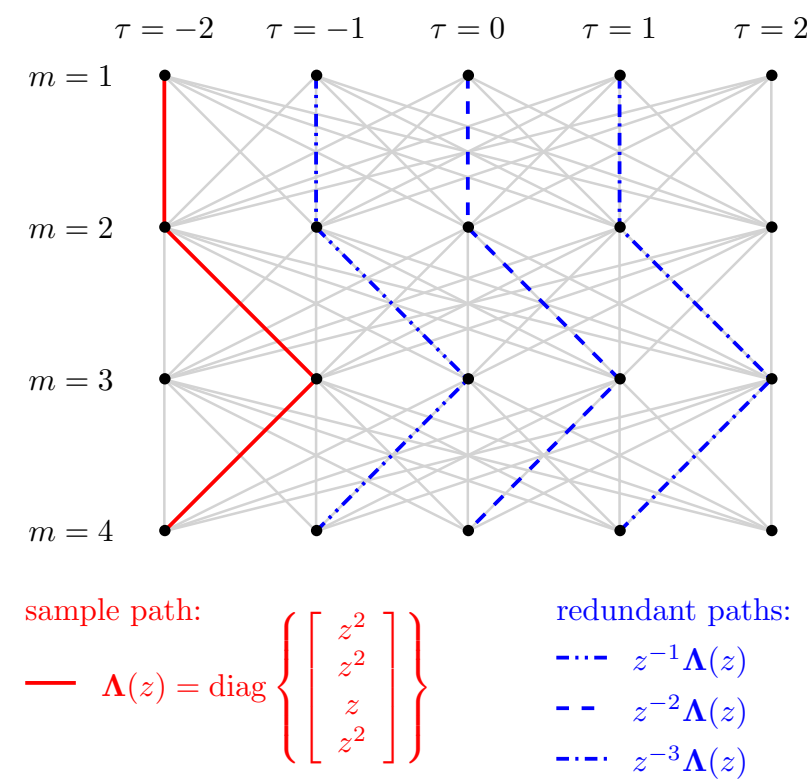

Fig. 1. Visualisation of possible delay matrices for $M=4$ and $L=1$; each top-bottom path in the $\left(2 \Delta_{\max }+1\right) \times M$ trellis defines the parameters of a possible delay matrix; one specific matrix is highlighted in red, with redundant matrices for horizontally shifted paths.

\section{B. Exhaustive Search}

In the case of a single shift algorithm, the maximum shift length, $\Delta_{\max }$, is $L$. Under multiple shifts the movement of one row/column pair will affect other rows and columns and so the maximum shift, $\Delta_{\max }$, becomes $\lceil(M-1) L / 2\rceil$.

Varying each diagonal element of $\boldsymbol{\Lambda}^{(i)}(z)$ in (11) over the interval $\tau \in\left[-\Delta_{\max }, \Delta_{\max }\right]$ will lead to $\left(2 \Delta_{\max }+1\right)^{M}$ possible shift combinations. This is visualised in Fig. 1 for the case $M=4$ and $L=1$, with the $m$ th row representing all possible values for $\tau^{(i, m)}$ in (11). Each path from top to bottom represents one particular combination of shifts, with a total number of $\left(2 \Delta_{\max }+1\right)^{M}$ possibilities. For larger values of $M$ or $L$, the diagram in Fig. 1 expands vertically or horizontally, respectively.

Of the established $\left(2 \Delta_{\max }+1\right)^{M}$ possible shift combinations, a number of combinations are redundant, because for (5), $z^{-\Delta} \Lambda^{(i)}(z)$, with $\Delta$ chosen arbitrarily, will implement a shift that is identical to $\boldsymbol{\Lambda}^{(i)}(z)$. As an example for the case of $\mathbf{S}^{(i-1)}[\tau] \in \mathbb{C}^{4 \times 4}$ with $L=1$, Fig. 1 highlights three redundant combinations that yield the same results as $\boldsymbol{\Lambda}^{(i)}(z)=\operatorname{diag}\left\{\left[\begin{array}{llll}z^{2} & z^{2} & z & z^{2}\end{array}\right]\right\}$. Selecting one path, its redundant copies can be identified by strict horizontal shifts within the trellis, which correspond to an overall delay or advance encapsulated by $z^{-\Delta}$.

Defining $\mathcal{S}$ as the set of shift combinations, the following theorem states its cardinality:

Theorem 1 (Cardinality of $\mathcal{S}$ ): For a parahermitian matrix $\boldsymbol{S}^{(i-1)}(z) \in \mathbb{C}^{M \times M}$ of order $2 L$, such that $\mathbf{S}^{(i-1)}[\tau]$ with a support of $2 L+1$, the set $\mathcal{S}$ of independent shift combinations that an exhaustive search algorithm has to evaluate has the cardinality

$$
|\mathcal{S}|=\left(2 \Delta_{\max }+1\right)^{M}-\left(2 \Delta_{\max }\right)^{M} .
$$

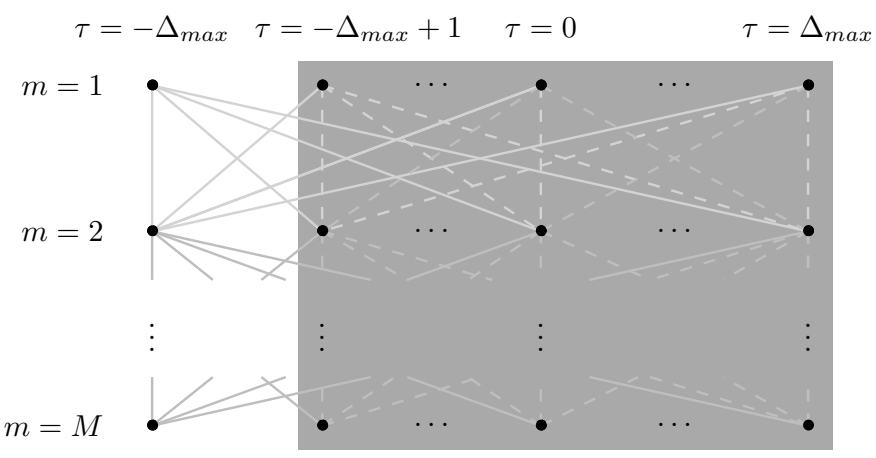

Fig. 2. Trellis of paths representing possible shift matrices. Redundant shift matrices according to the definition of (14), which do not involve the left-most column with $\tau=-L$, have $\left(2 \Delta_{\max }\right)^{M}$ paths confined to the shaded area.

Proof: We define a redundant delay matrix $\Gamma^{(i)}(z)$ as one that can be obtained by delaying a genuine delay matrix $\Lambda^{(i)}(z) \in \mathcal{S}$, such that $\Gamma^{(i)}(z)=z^{-\Delta} \Lambda^{(i)}(z)$ with $\Delta>0$. W.r.t. the sample trellis in Fig. 1, the path belonging to the parameter set of $\boldsymbol{\Lambda}^{(i)}(z)$ has to include at least one node in the left-most column of the trellis, i.e. if $\boldsymbol{\Lambda}^{(i)}(z)$ is constructed according to (11),

$$
\Lambda^{(i)}(z) \in \mathcal{S} \longleftrightarrow \min _{m} \tau^{(i, m)}=-\Delta_{\max } .
$$

Therefore, a redundant matrix $\Gamma^{(i)}(z)-$ also constructed according to (11) - is characterised by not reaching any node in the left-most column, or

$$
\Gamma^{(i)}(z) \notin \mathcal{S} \longleftrightarrow \min _{m} \tau^{(i, m)}>-\Delta_{\max } .
$$

Since any path fulfilling (14) has to lie entirely within the shaded area in Fig. 2, occupying the $2 \Delta_{\max }$ left-most columns of the trellis, there are $\left(2 \Delta_{\max }\right)^{M}$ paths belonging to redundant delay matrices. With a total possibility of $\left(2 \Delta_{\max }+1\right)^{M}$ combinations within the trellis, (12) is proven.

The cardinality $|\mathcal{S}|$ in (12) is important, as it restricts the exhaustive search and therefore limits its implementation cost. The definition of an independent delay matrix according to (13) is somewhat arbitrary, and its place in the set $\mathcal{S}$ could also be taken by one of its redundant copies $z^{-\Delta} \boldsymbol{\Lambda}^{(i)}(z)$. This has no impact on $\boldsymbol{S}^{(i) \prime}(z)$ in (5) and therefore does not affect $\boldsymbol{D}(z)$ in (7). However, selecting a copy with minimum degree in $z^{-1}$ will influence the order of the paraunitary matrix $\boldsymbol{Q}(z)$ in (8), and a search algorithm would therefore have to operate with care to either keep the order increase low w.r.t. $Q(z)$, or to ensure that any growth by trailing zero matrices is curtailed.

The size of the search space for delay matrices applicable to $\mathbf{S}^{(i-1)}[\tau] \in \mathbb{C}^{M \times M}$ is shown in Fig. 3 for the cases $M=$ $3,5,7$ and over a range of maximum lag values $L$. Both the number of possible as well as the independent number of shifts - those belonging to $\mathcal{S}$ - are detailed.

\section{Convergence}

Theorem 2 (Maximum Energy SMD Algorithm): With a sufficiently high number of iterations, $I$, the maximum energy SMD algorithm, defined as transferring maximal energy into the off-diagonal elements of $\boldsymbol{S}^{(i \prime)}[0]$ and subsequently onto 


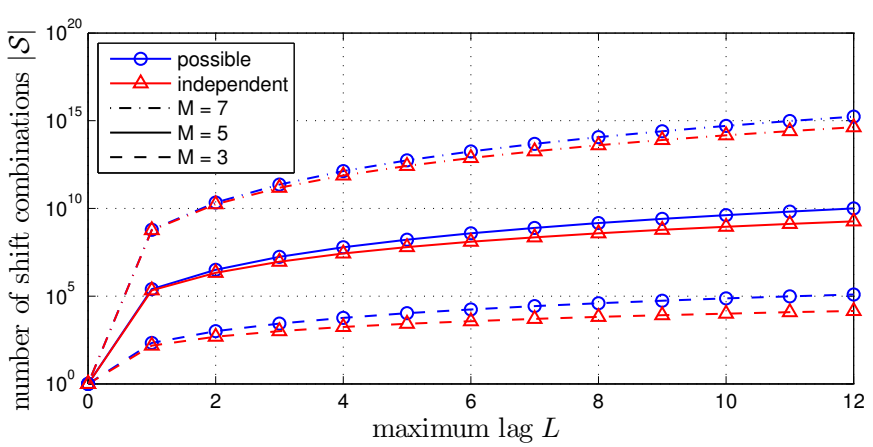

Fig. 3. Number of shift combinations that an exhaustive search algorithm has to evaluate for $\boldsymbol{S}^{(i-1)}[\tau] \in \mathbb{C}^{M \times M}$ for $M=3,5,7$ with support $2 L+1$.

the diagonal of $\boldsymbol{S}^{(i)}[0]$ at every iteration $i$, will converge to an arbitrarily low limit $\epsilon$ for the off-diagonal energy.

Proof: Since the exhaustive search yields a transfer of at least as much off-diagonal energy per iteration as MSMESMD, Theorem 2 is covered by the proof in [11]. The value of $\epsilon$ is determined by the stopping criterion of the algorithm.

\section{Implementation}

The implemented algorithm first finds the list of all $\left(2 \Delta_{\max }+1\right)^{M}$ possible shifts, which is then pruned to remove the redundant $\left(2 \Delta_{\max }\right)^{M}$ delay operations to obtain $\mathcal{S}$. Within $\mathcal{S}$, the best possible shift combination for $\mathbf{S}^{(i-1)}[\tau] \in \mathbb{C}^{M \times M}$ with support $2 L+1$ is then identified.

According to Fig. 3, the complexity of the exhaustive search grows very fast for an increase in the spatial matrix dimension $M$, but also a rise in the lag dimension $L$ has significant impact. In general the complexity is such that it is not possible to calculate a full maximum energy SMD algorithm, as it may reach large values of $L$ during its iteration. Therefore, we will below only analyse the exhaustive search approach for a single iteration step, but use the results as a benchmark for other SMD algorithms based on limited search strategies.

\section{RESUlTS}

The comparison of search algorithms is calculated over an ensemble of 1000 realisations of a parahermitian matrix $\boldsymbol{S}(z)=\boldsymbol{A}(z) \tilde{\boldsymbol{A}}(z)$, which are obtained from a matrix $\boldsymbol{A}(z) \in$ $\mathbb{C}^{M \times M}$ of order $L$ with independent and identically distributed Gaussian elements. First, this section looks at a single iteration of SMD algorithms as reviewed in Sec. II, using the maximum energy SMD of Sec. III as a benchmark, before the general approximation of a PEVD is investigated.

\section{A. Energy Transfer}

Simulating the $i$ th iteration of an SMD algorithm, we set $\boldsymbol{S}^{(i-1)}(z)=\boldsymbol{S}(z)$ but with zeroed off-diagonal elements in $\mathbf{S}^{(i-1)}[0]$. The performance of the various search algorithms is evaluated by calculating the off-diagonal energy of the zero $\operatorname{lag} \mathbf{S}^{(i \prime)}[0]$ after shifts have been performed, i.e.

$$
E=\sum_{k=1}^{M}\left\|\hat{\mathbf{s}}_{k}^{(i \prime)}[0]\right\|_{2} \quad .
$$

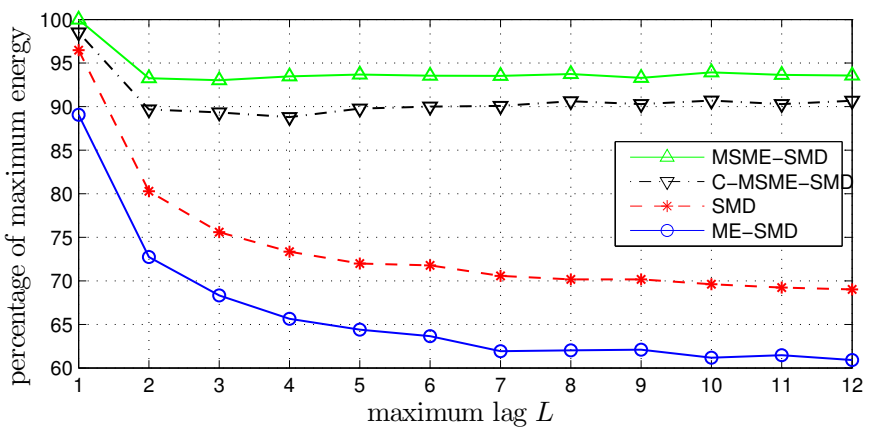

Fig. 4. Percentage of maximum off-diagonal energy transferred by SMD algorithms in the $i$ th iteration for $\boldsymbol{S}^{(i)}(z) \in \mathbb{C}^{3 \times 3}$ for different maximum lag values $L$.

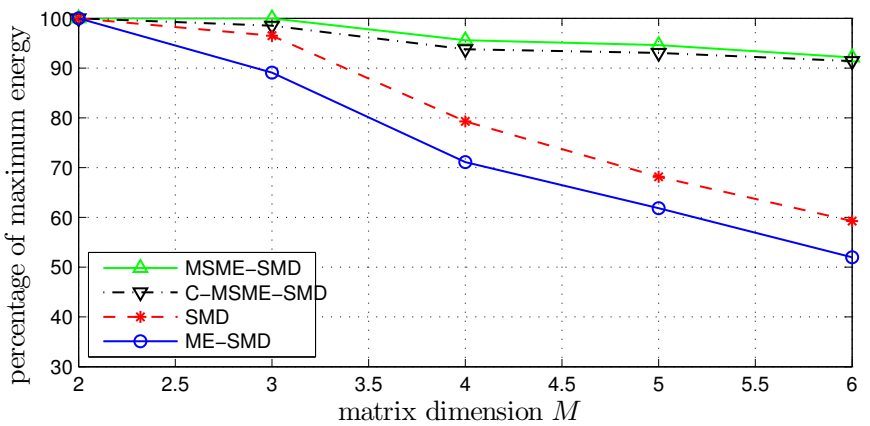

Fig. 5. Percentage of maximum off-diagonal energy transferred by SMD algorithms for $\boldsymbol{S}^{(i-1)}(z) \in \mathbb{C}^{M \times M}$ with $L=1$ for variable $M$.

Fig. 4 compares the values of $E$ normalised by the result of the maximum energy SMD algorithm for $M=3$ over a range of values for the maximum lag $L$. The same situation is shown in Fig. 5, but for a fixed value of $L=1$, the matrix dimension $M$ is varied.

The matrix dimension $M$ has a significant impact on the optimality of the energy transfer of SMD algorithms that only shift a single column per iteration, i.e. the standard SMD and the ME-SMD algorithms as defined in Secs. II-B and II-C. The latter methods drop performance quickly in Fig. 5, the for larger values of $M$ and only a single column to shift, their potential for transferring off-diagonal energy rapidly declines. Also an increase in the lag dimension in Fig. 4 affects these algorithms, although the impact of $L$ is not as severe as $M$, and seems to approximately level out for larger values of $L$.

The multiple shift SMD versions detailed in Sec. II-D are only modestly affected by the maximum lag $L$, and show a slight decline with an increase in matrix dimensions. For the values investigated however, both MSME-SMD and CMSME-SMD transfer on average $90 \%$ of the energy that is maximally attainable with an exhaustive search.

\section{B. Order Increase}

The shift matrices $\Lambda^{(i)}(z)$ obtained by the various SMD algorithms increase the order of the parahermitian and paraunitary matrices via (7) and (8). It can be seen that the advantage in energy transfer of SMD and ME-SMD comes at the expense of a larger growth in order [11]. Fig. 6 shows the average growth in length of the paraunitary matrix during the $i$ th 


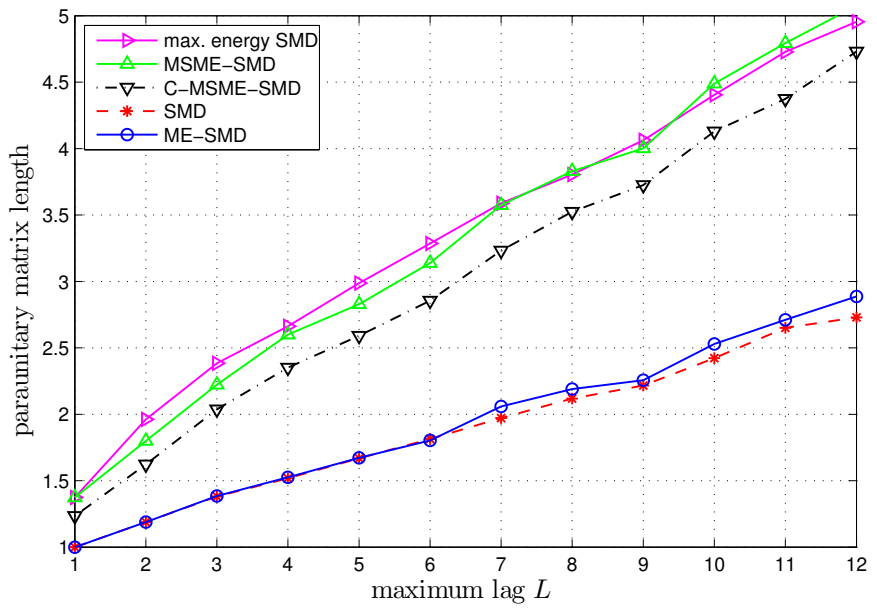

Fig. 6. Length growth of paraunitary matrix during the $i$ th iteration for $\boldsymbol{S}^{(i-1)}(z) \in \mathbb{C}^{M \times M}$ with maximum lag $L=1$ and variable $M$.

iteration, where it is interesting to note that despite its higher transfer of energy, the maximum energy SMD algorithm does not cause a greater growth than either MSME-SMD or CMSME-SMD.

\section{Diagonalisation}

Based on the ensemble of parahermitian matrices $\boldsymbol{S}(z)$ defined at the beginning of Sec. IV, the diagonalisation performance for $\boldsymbol{R}(z)=\boldsymbol{S}(z)$ with $M=L=5$ over a number of iterations $i$ is provided in Fig. 7. The graph shows the remaining off-diagonal energy, normalised by the total energy in $\boldsymbol{R}(z)$ which is invariance under paraunitary operations. With the maximum energy SMD too complex to obtain ensemble averaged results, only the SMD algorithms reviewed in Sec. II and SBR2 [2] are shown. Since the multiple-shift versions perform within approx. $10 \%$ of the maximum transferable off-diagonal energy per iteration, these limited search-strategy algorithm provide an excellent tradeoff between energy transfer and implementation complexity.

\section{CONCLUSiON}

To iteratively approximate a PEVD with high accuracy, this paper has presented a maximum energy approach for sequential matrix diagonalisation which transfers the maximally achievable off-diagonal power in very iteration step. This has required an exhaustive search over all independent shift combinations in the SMD algorithm per iteration. The overall size of the search space has been determined, which increases dramatically as the parahermitian matrix grows both in terms of space and lag dimension. Although the resulting complexity does not permit the maximum energy SMD algorithm to calculate a full iterative PEVD, the performance over one iteration provides a valuable benchmark for other SMD algorithms.

On average, for the parameter set considered, it has been demonstrated that recently introduced multiple-shift SMD algorithms, which optimise energy over a small, limited search space, can perform within $10 \%$ of the transferred energy of the maximum energy SMD approach. Therefore, multiple-shift

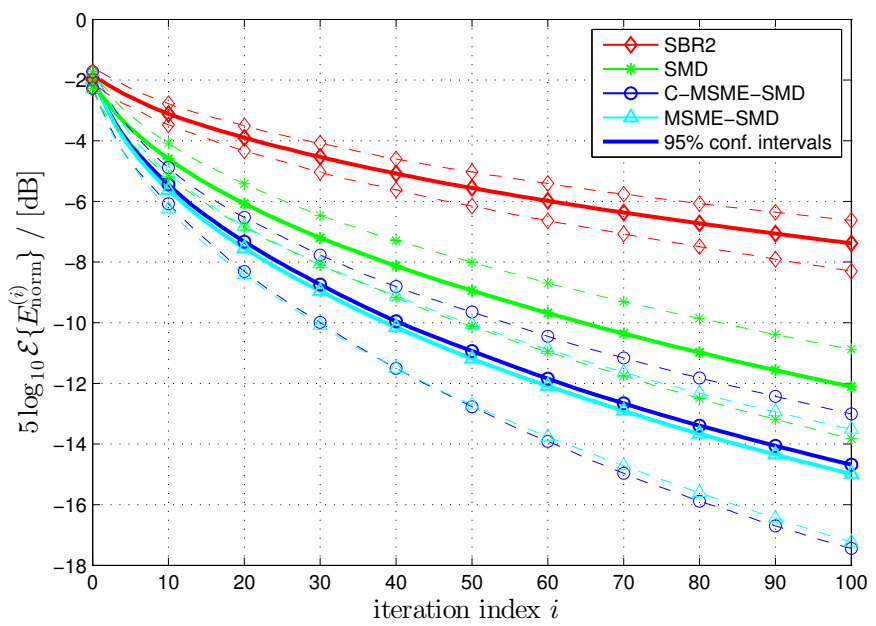

Fig. 7. Diagonalisation performance of SBR2 [2] and various SMD algorithms [10], [11], measured by the remaining off-diaginal energy normalised by the total energy in the parahermitian matrix.

SMD algorithms appear to perform close to the optimum and provide a very good trade-off between the loss of performance and the reduction in computational complexity when compared to maximum energy SMD.

\section{ACKNOWLEDGEMENT}

This work was supported by the Engineering and Physical Sciences Research Council (EPSRC) Grant number EP/K014307/1 and the MOD University Defence Research Collaboration in Signal Processing.

\section{REFERENCES}

[1] P. P. Vaidyanathan. Multirate Systems and Filter Banks. Prentice Hall, Englewood Cliffs, 1993.

[2] J. G. McWhirter, P. D. Baxter, T. Cooper, S. Redif, and J. Foster. An EVD Algorithm for Para-Hermitian Polynomial Matrices. IEEE Trans SP, 55(5):2158-2169, May 2007.

[3] G. H. Golub and C. F. Van Loan. Matrix Computations. John Hopkins University Press, Baltimore, Maryland, 1996.

[4] S. Icart and P. Comon. Some properties of Laurent polynomial matrices. In 9th IMA Conf. on Maths in SP, Birmingham, UK, Dec. 2012.

[5] S. Redif, J. McWhirter, and S. Weiss. Design of FIR paraunitary filter banks for subband coding using a polynomial eigenvalue decomposition. IEEE Trans SP, 59(11):5253-5264, Nov. 2011.

[6] S. Weiss, S. Redif, T. Cooper, C. Liu, P. D. Baxter, and J. G. McWhirter. Paraunitary Oversampled Filter Bank Design for Channel Coding. EURASIP Journal of Applied SP, 2006.

[7] C. H. Ta and S. Weiss. A Design of Precoding and Equalisation for Broadband MIMO Systems. In 15th Int. Conf. DSP, pp. 571-574, Cardiff, UK, July 2007.

[8] M. Alrmah, S. Weiss, and S. Lambotharan. An extension of the music algorithm to broadband scenarios using polynomial eigenvalue decomposition. In EURASIP, pages 629-633, Barcelona, Spain, Aug. 2011.

[9] A. Tkacenko. Approximate eigenvalue decomposition of para-hermitian systems through successive fir paraunitary transformations. In IEEE ICASSP, pp. 4074-4077, Dallas, TX, Mar. 2010.

[10] S. Redif, S. Weiss, and J. McWhirter. Sequential Matrix Diagonalisation Algorithms for Polynomial EVD of Parahermitian Matrices. IEEE Trans $S P$, to appear.

[11] J. Corr, K. Thompson, S. Weiss, J. McWhirter, S. Redif, and I. Proulder. Multiple shift maximum element sequential matrix diagonalisation for parahermitian matrices. In IEEE SSP Workshop, Gold Coast, Australia, June 2014.

[12] J. Corr, K. Thompson, S. Weiss, J. G. McWhirter, and I. K. Proudler. Causality-Constrained multiple shift sequential matrix diagonalisation for parahermitian matrices. In EUSIPCO, Lisbon, Portugal, Sep. 2014. 